\title{
Loss of p16(INK4a) is associated with reduced patient survival in soft tissue tumours, and indicates a senescence barrier
}

\author{
Thomas Knösel, ${ }_{1}^{1}$ Annelore Altendorf-Hofmann, ${ }^{2}$ Lars Lindner, ${ }^{3}$ Rolf Issels, ${ }^{3}$ \\ Heiko Hermeking, ${ }^{4}$ Gesa Schuebbe, ${ }^{3}$ Sebastian Gibis, ${ }^{3}$ Helge Siemens, ${ }^{4}$ \\ Eric Kampmann, ${ }^{3}$ Thomas Kirchner ${ }^{1}$
}

Additional material is published online only. To view please visit the journal online (http://dx.doi.org/10.1136/ jclinpath-2013-202106).

1 Institute of Pathology, Ludwig-Maximilians-University (LMU), Munich, Germany 2Department of General, Visceral und Vascular Surgery, Friedrich-Schiller University, Jena, Germany

${ }^{3}$ Department of Internal Medicine III, LudwigMaximilians-University (LMU), Munich, Germany ${ }^{4}$ Experimental and Molecular Pathology, Institute of Pathology, LudwigMaximilians-University (LMU), Munich, Germany

\section{Correspondence to} Professor Thomas Knösel, Institute of Pathology, LudwigMaximilians-University (LMU), Thalkirchnerstr. 36, Munich 80337, Germany;

Thomas.Knoesel@med. uni-muenchen.de

Received 10 December 2013 Revised 26 March 2014 Accepted 31 March 2014 Published Online First 19 April 2014

\section{ABSTRACT}

Aims p16(INK4a) is an important factor in

carcinogenesis, and its expression is linked to oncogeneinduced senescence. Very recently it was shown that upregulation and downregulation of p16 indicates a senescence barrier in the serrated route of colorectal cancer. However, in soft tissue sarcoma (STS), the senescence mechanism is still not understood. In this study, we analysed a well characterised cohort of STS for p16(INK4a) expression and correlated the results with clinicopathological parameters including survival.

Methods Tissue microarrays (TMA) of 183 soft tissue and bone tumours were analysed immunohistochemically. Furthermore, mRNA expression of p16(INK4a) was evaluated in four sarcoma cell lines, and a demethylation test was performed by treatment with 5 -aza- $2^{\prime}$ -

deoxycytide.

Results On protein level, expression of p16(INK4a) was observed in undifferentiated pleomorphic sarcoma (UPS) in $69.1 \%$, leiomyosarcoma in $85.7 \%$, synovial sarcoma in $77.8 \%$, liposarcoma in $88.9 \%$, angiosarcoma in $60.9 \%$ and MPNST in $22.2 \%$. Loss of p16(INK4a) was observed in high grade sarcomas and showed a significant correlation with reduced patient survival $(p=0.032)$. On DNA level, one out of four sarcoma cell lines exhibited a methylated p16(INK4a) promoter analysed by methylation-specific PCR. p16(INK4a) mRNA and protein expression was restored after demethylation using 5-aza2'-deoxycytide.

Conclusions Upregulation of p16(INK4a) might be associated with the induction of senescence and indicates a senescence barrier. Downregulation of p16(INK4a) is found in malignant progression, and is significantly correlated with reduced patient survival. Downregulation of p16(INK4a) may be explained by DNAhypermethylation in sarcoma cells.

\section{INTRODUCTION}

Soft tissue sarcomas (STS) are a rare, heterogeneous and highly malignant group of tumours originating from the mesenchymal lineage. Local recurrence is common (20\%), and metastases occur in one-third of the patients. ${ }^{1} 2$ Prognostic and predictive markers in potentially curable STS should guide therapy after surgical resection. Neoadjuvant therapy is used with increasing frequency and may improve prognosis in high-risk cases. The indication for adjuvant chemotherapy for high-risk patients is still under debate, therefore, new diagnostic, predictive and prognostic biomarkers are needed. ${ }^{3}$ These biomarkers should support the clinical treatment of the neoplastic process, for example, by selecting specific drug regiments.

p16(INK4a) is a cell-cycle inhibitor and an important factor in carcinogenesis. It controls p16INK4a-dependent growth arrest and its expression can be linked to oncogene-induced senescence. ${ }^{4-6}$ Oncogene-induced senescence is characterised by an irreversible growth arrest and occurs as a consequence of oncogene activation. The abruption of oncogene-induced senescence is an important mechanism for malignant transformation and can be caused by genetic alterations leading to a loss of function of p16(INK4a). The induction of p16 (INK4a) in premalignant lesions, and its loss during malignant transformation, have been demonstrated in the carcinogenesis of the liver, lung and pancreas. $^{7-9}$ Loss of p16(INK4a) is often caused by CDKN2A p16(INK4a) promoter hypermethylation. ${ }^{10}$ However, in sarcoma genesis, the senescence mechanism is still not understood and has not been described before. In our previous studies, we showed that upregulation and downregulation of p16(INK4a) expression in BRAF-mutated polyps/ adenomas indicates a senescence barrier in the serrated route to colonic cancer. ${ }^{6}$ In this study, we expanded the analysis to different types of sarcomas in order to explore mechanisms of senescence and to find associations with clinicopathological parameters that may be important for tumour progression and survival. We analysed the expression of p16(INK4a) in a well-characterised cohort of sarcomas, and investigated the methylation status of the p16(INK4a) promoter in cell lines representing different sarcoma types.

Our objectives in this study were (1) to evaluate the p16(INK4a) expression status in a series of wellcharacterised sarcomas on protein level, (2) to correlate the expression with clinicopathological data including patient survival, and (3) to analyse the methylation status in sarcoma cell lines to detect potential pathomechanisms in sarcomagenesis.

\section{MATERIALS AND METHODS}

\section{Patients}

TMAs containing samples from 183 patients were constructed. Tissue samples mostly originated from surgical specimens of patients diagnosed and treated from 1989 to 2012 in the Sarcoma Centre at Ludwig-Maximilians University, Munich. Data on clinical parameters, including sex, age and 
primary site were extracted from the pathologists` original reports and the database of (ClinicalTrials.gov), number NCT $00003052 .{ }^{11}$ In addition to the original pathology reports, microscopic findings (tumour type, according to current WHO classification and degree of differentiation) were reassessed by two authors (EK and TK). Nearly all patients underwent surgical resection of the tumour, and all but $18(9,8 \%)$ patients had additional radiotherapy. The patients' age at the time of diagnosis ranged from 19 to 81 years (median age 54 years, female $\mathrm{n}=101$, male $\mathrm{n}=82$ ). The sarcomas were located in the extremities in 60, and in non-extremities in 123 cases.

\section{Tissue microarray construction}

The sarcoma TMAs were assembled using $0.6 \mathrm{~mm}$ punch biopsies in duplicate from all 183 samples according to standard procedure. ${ }^{12}$ Totally, 183 specimens of sarcoma tissue were evaluated.

\section{Immunhistochemistry}

Immunohistochemical staining was done on $5 \mu \mathrm{m}$ tissue sections of formalin-fixed paraffin-embedded tumour samples. As primary antibodies, anti-p16Ink4a mouse monoclonal antibody (Diagnostic Biosystems, dilution $1: 100$ ) was used. Staining was performed on a Ventana Benchmark XT autostainer with the XT ultraView DAB Kit (Ventana Medical Systems). All slides were counterstained with Haematoxylin (Vector). To exclude unspecific staining, system controls were included. As positive controls for the immunohistochemistry, cervical intraepithelial neoplasia (p16INK4a) was used. The immunostaining of the cells was evaluated and scored semiquantitatively: 0 , negative; 1 , weak; 2 , moderate and 3 , strongly positive.

\section{Statistical analysis}

Follow-up was performed in outpatient clinics or by contacting the patients' general practitioners, and data were updated until September 2012. Survival was calculated from the date of first diagnosis. Statistical analyses were performed by SPSS 20.0 (SPSS, Chicago, IL) software. Survival curves were calculated by the Kaplan-Meier method. The log rank test was used to assess differences in survival. The HRs are given with CIs for each factor.

\section{Cell lines and treatment}

Human sarcoma cell lines SW872 and SW982 were grown in DMEM (Invitrogen) supplemented with 5\% FCS. The human sarcoma cell line SKUT1 was kept in MEM supplemented with $10 \%$ FCS, 1\% NEAA and 1\% L-Glutamine. Human sarcoma cell line U2OS was kept in DMEM supplemented with 10\% FCS. All cells were incubated at $5 \% \mathrm{CO}_{2}$ according to standard procedures.

U2OS cells were treated by adding the de-methylating agent 5 -aza-2'deoxycytidine (5-Aza, Sigma) for $72 \mathrm{~h}$ to the medium. Different concentrations $(0,1$ and $10 \mu \mathrm{M})$ were tested.

\section{DNA isolation and bisulfite treatment}

Genomic DNA was isolated using the DNeasy Blood \& Tissue Kit (Qiagen) according to the manufacturer's instructions. Subsequently, $500 \mathrm{ng}$ of DNA was treated with bisulphite using the EZ DNA Methylation Kit (Zymo Research).

\section{Methylation specific PCR (MSP)}

Methylation-specific PCR analyses were carried out in a total volume of $20 \mu \mathrm{l}$ using 1.5 units Platinum Taq-polymerase (Invitrogen) per reaction, and $\sim 50 \mathrm{ng}$ of bisulphite converted
DNA as template. The reaction conditions were the following: $95^{\circ} \mathrm{C}$ for $5 \mathrm{~min}$, followed by 35 cycles of $95^{\circ} \mathrm{C}$ for $30 \mathrm{~s}, 65^{\circ} \mathrm{C}$ for $30 \mathrm{~s}$ and $72^{\circ} \mathrm{C}$ for $30 \mathrm{~s}$. The final elongation step was performed for $7 \mathrm{~min}$ at $72^{\circ} \mathrm{C}$. The oligonucleotide sequences used for the MSP are the following:

p16M fwd: 5'-TTATTAGAGGGTGGGGCGGATCGC-3';
p16M rev: 5'-GACCCCGAACCGCGACCGTAA -3';
p16U fwd: 5'- TTATTAGAGGGTGGGGTGGATTGT -3';
p16U rev: 5' - CAACCCCAAACCACAACCATAA -3'.

Amplified fragments $(M=150 \mathrm{bp}, \mathrm{U}=151 \mathrm{bp})$ were separated by electrophoresis on $10 \%$ polyacrylamide gels, and visualised by staining with ethidium bromide. Pictures were taken using a Multilmage Light Cabinet (Alpha Innotech).

\section{Western blot analysis}

SDS-PAGE and western blotting were performed according to standard protocols. Cells were lysed in RIPA lysis buffer $(50 \mathrm{mM}$ Tris/HCl, pH 8.0, $250 \mathrm{mM} \mathrm{NaCl}, 1 \% \mathrm{NP} 40,0.5 \%$ (w/v) sodium deoxycholate, $0.1 \%$ sodium dodecylsulfate, and complete mini protease inhibitor tablets (Roche)). Lysates were sonicated and centrifuged at $16.060 \mathrm{~g}$ for $15 \mathrm{~min}$ at $4^{\circ} \mathrm{C}$. Per lane, $60 \mu \mathrm{g}$ of whole cell lysate was separated using a $12 \%$ SDS-acrylamide gel and transferred on Immobilon PVDF membranes (Millipore). ECL signals were recorded using a CF440 Imager (Kodak). Antibodies were the following:

$\alpha$-tubulin: monoclonal anti- $\alpha$-tubulin, clone DM1A (Sigma)

p16: monoclonal (F-12) p16 antibody, (Santa Cruz)

\section{Quantitative real-time PCR}

Total RNA was prepared with the High Pure RNA Isolation Kit (Roche) according to the manufacturer's instructions. cDNA was generated from $1 \mu \mathrm{g}$ of total RNA per sample using anchored oligo(dT) primers (Verso Kit). RT-qPCR was performed by using the LightCycler 480 (Roche) and the Fast SYBR Green Master Mix (Applied Biosystems). Only primer pairs resulting in a single peak in the melting curve analysis were used. The qPCR-primers used in this study were the following:

GAPDH qPCR fwd: 5'- GCTCTCTGCTCCTCCTGTTC-3'; GAPDH qPCR rev: 5' - ACGACCAAATCCGTTGACTC-3; p16 qPCR fwd: 5' - CAACGCACCGAATAGTTACG-3; p16 qPCR rev: 5' - CTGCCCATCATCATGACCTGG-3;

\section{RESULTS}

\section{Immunohistochemistry}

The results of the entire tumour collective are summarised in table 1 . The expression was scored semiquantitatively by a 4-tier scale ( 0 : negative; 1 : weak; 2 : moderate; 3 : strongly positive; figure 1$)$ that was reduced to a 2 -tier system ( 0 : negative; vs $1 / 2 / 3$ : positive) for statistical analysis. On protein level, positive expression was observed in $136(74.3 \%)$ specimens. In detail, the ratio of positive expression according to the different tumour types is given in table 1 .

\section{Immunohistochemistry and clinicopathological parameters}

Percentages of positive expression were calculated for age, sex and tumour site (extremities vs non-extremities); 70\% $(0.5886$; $0.7842)$ of male specimens and $78 \%(0.6922 ; 0.8515)$ of female specimens showed positive staining with $\mathrm{p} 16$. No statistical significant correlation with protein expression could be demonstrated.

Grade 1, grade 2 and grade 3 tumours were observed in $3.3 \%, 34.4 \%$ and $62.3 \%$, respectively. $100 \%(0.4385 ; 1)$ of 
Table 1 Expression of p16 according to the different entities

\begin{tabular}{lrrrl}
\hline & \multicolumn{5}{c}{$\mathbf{P} \mathbf{1 6}$ positive } \\
\cline { 3 - 5 } Entity & All & $\mathbf{n}$ & $\%$ & $\mathbf{9 5 \%} \mathrm{Cl}$ \\
\hline UPS & 55 & 38 & 69.1 & $(0.5597$ to 0.7972$)$ \\
Leiomyosarcoma & 35 & 30 & 85.7 & $(0.7062$ to 0.9374$)$ \\
Liposarcoma & 27 & 24 & 88.9 & $(0.7194$ to 0.9615$)$ \\
Angiosarcoma & 23 & 14 & 60.9 & $(0.4079$ to 0.7784$)$ \\
Synovialsarcoma & 18 & 14 & 77.8 & $(0.5478$ to 0.91$)$ \\
MPNST & 9 & 2 & 22.2 & $(0.0632$ to 0.5474$)$ \\
Carcinosarcoma & 4 & 4 & 100 & $(0.5101$ to 1$)$ \\
Chondrosarcoma & 3 & 2 & 67 & $(0.2077$ to 0.9385$)$ \\
Rhabdomyosarcoma & 3 & 3 & 100 & $(0.4385$ to 1$)$ \\
Malignant SFT & 2 & 2 & 100 & $(0.3424$ to 1$)$ \\
Alveolar soft tissue sarcoma & 2 & 2 & 100 & $(0.3424$ to 1$)$ \\
Myxofibrosarcoma & 2 & 1 & 50 & $(0.0945$ to 0.9055$)$ \\
All patients & 183 & 136 & 74.3 & $(0.6753$ to 0.801$)$ \\
\hline
\end{tabular}

grade 1 tumours showed positive expression of $\mathrm{p} 16$. In grade 2 and grade 3 tumours, nearly equal percentages of positive staining $78.0 \%(0.6476 ; 0.8725)$ vs $74.7 \%(0.6379 ; 0.8314))$ were observed.

\section{Survival analysis}

At the time of last follow-up, 70 patients were dead and 113 were alive. The median survival time for the whole group was
$77(41 ; 99)$ months, with a 5 -year and 10 -year survival rate of $56 \%$ and $46 \%$, respectively.

High-grade tumours were subdivided according to tumour type. Survival was calculated for groups containing at least 15 patients; 10-year survival rates were similar for liposarcomas, leiomyosarcomas, synovial sarcoma and UPS (60\%, 55\%, 54\%, and $43 \%$, respectively), but statistically significantly lower for angiosarcomas (11\%, figure 2).

Kaplan-Meier analysis of all patients showed a significantly higher survival rate for $\mathrm{p} 16$-positive tumours compared to p16-negative specimens $(p=0.032)$. The same result was seen for leiomyosarcomas, liposarcomas and synovial sarcomas. No statistical significant difference in survival rates depending on p16 expression was seen in UPS and angiosarcomas (table 2).

\section{Investigation of the p16 methylation status in sarcoma cell lines}

The methylation status of $p 16(I N K 4 a)$ promotor was analysed in the sarcoma cell lines SW872 (liposarcoma), SW982 (synovial sarcoma), SKUT1 (leiomyosarcoma) and U2OS (osteosarcoma). For this, genomic DNA was isolated, treated with bisulphite and applied as template for subsequent methylation-specific PCR (MSP). While the MSP for SKUT1 cells did not result in a detectable product, SW872 and SW982 showed a band for unmethylated $p 16$, whereas for U2OS cells, a band representing methylated $p 16$ was observed (figure $3 \mathrm{~A}$ ). Water was used in a non-template negative control, and gDNA of the colorectal cancer cell line HCT116 was used as positive control.
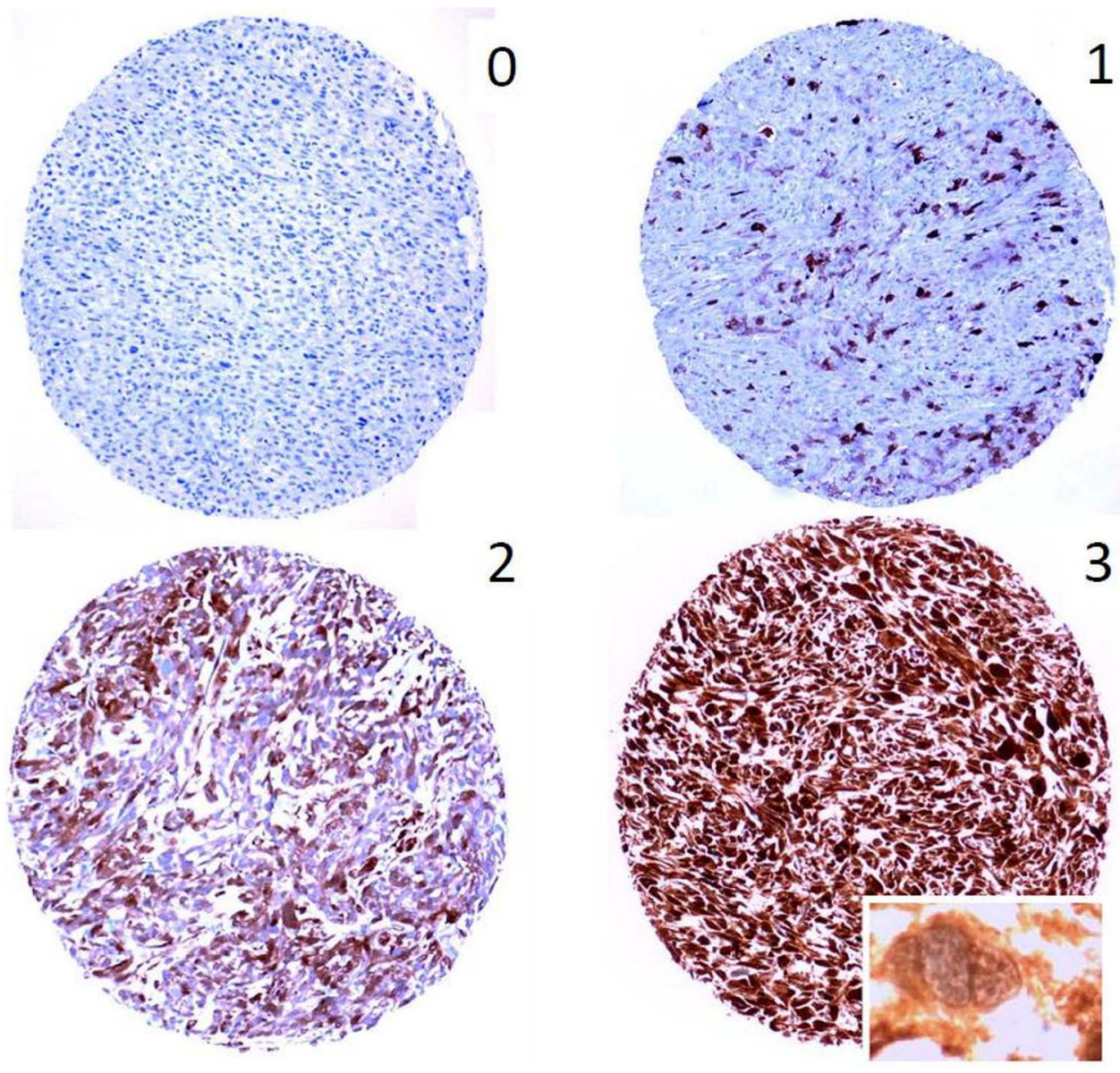

Figure 1 Examples of the immunohistochemical assessment of p16(INK4a) staining in sarcomas. Negative staining of tumour cells (0), weakly positive (1), moderately positive (2), strongly positive (3) (10xmagnification, insert: 40xmagnification, nuclear and cytoplasmatic staining). 


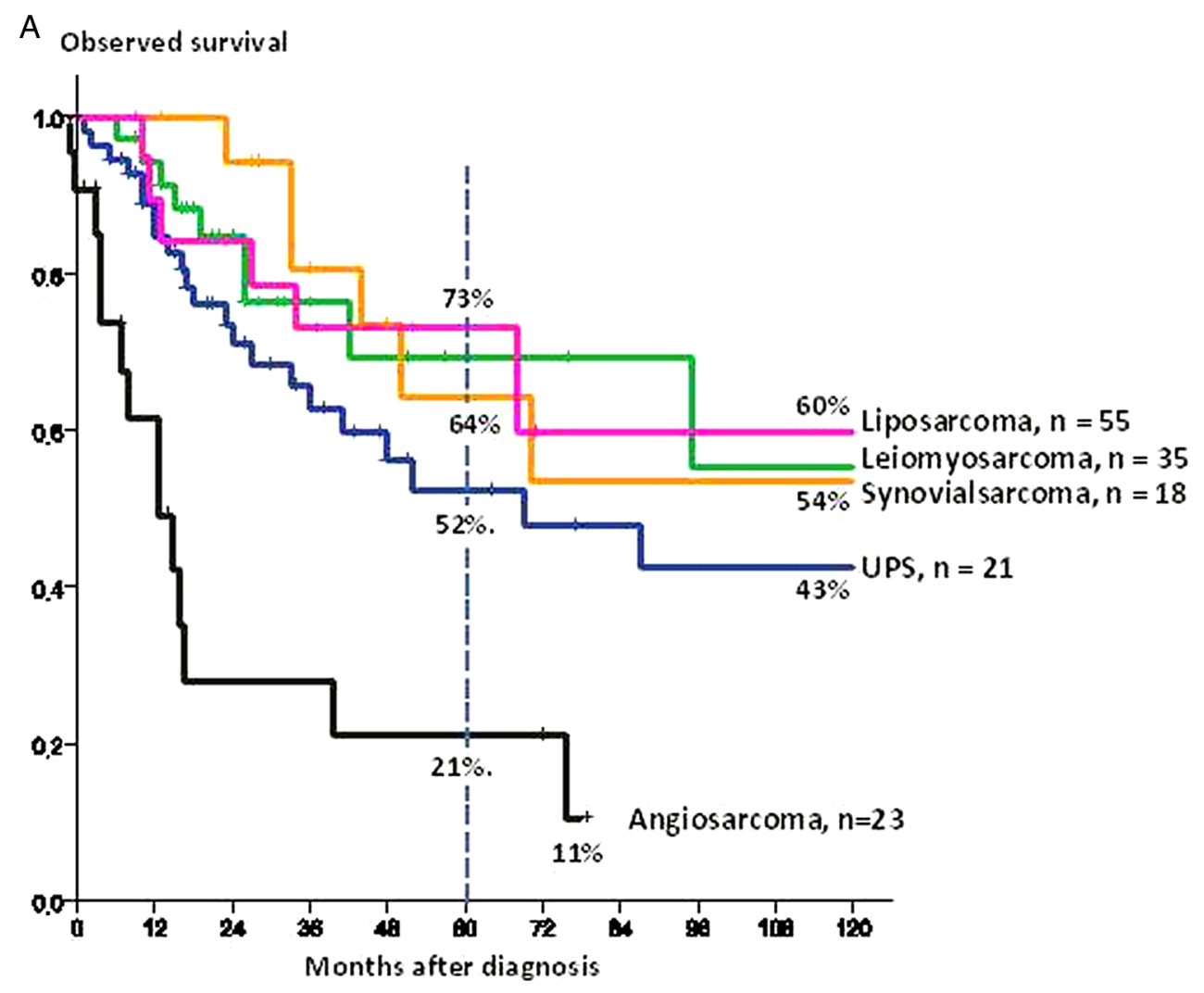

B

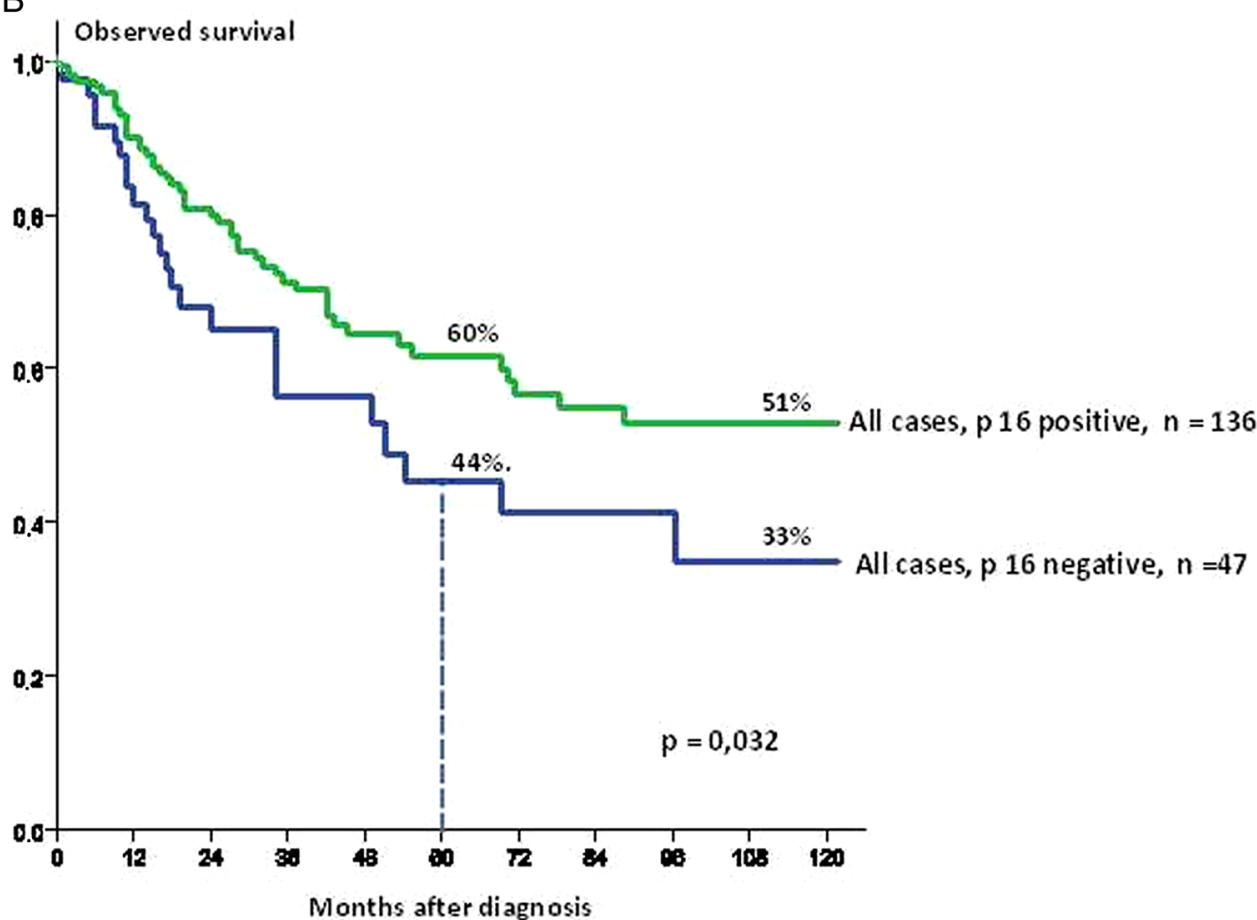

Figure 2 Univariate analysis (log-rank test, Kaplan-Meier curves) (A) of the different sarcoma entities and (B) expression of p16(INK4a) with observed survival $(p=0.032)$.

In order to test whether this methylation was reversible upon use of a demethylating agent, we exposed U2OS cells to different concentrations of the pharmacological substance 5-aza$2^{\prime}$ deoxycytidine (5-Aza). Indeed, following treatment with 1, 10 or $50 \mu \mathrm{M} \mathrm{5-Aza} \mathrm{for} 72 \mathrm{~h}$, a band for unmethylated p16 was restored, as shown by MSP (figure 3B). To test, whether demethylation of the gene resulted in an increased expression of the $p 16$ mRNA, we isolated RNA from cells treated with $10 \mu \mathrm{M} 5$-Aza, or left untreated, and included the RNA of the cervix carcinoma cell line HeLa as a positive control. While no p16 was detectable in untreated U2OS cells, expression was significantly induced upon treatment (figure 3C). 
Table 2 Differences in observed survival depending on expression of p16 $(\mathrm{HR}, \mathrm{Cl}=95 \% \mathrm{Cl})$

\begin{tabular}{lclll}
\hline Entity & Patients & HR & $95 \%$ Cl & p Value \\
\hline All & 183 & 1.654 & 1.028 to 2.660 & 0.038 \\
UPS & 55 & 0.452 & 0.273 to 1.784 & 0.452 \\
Leiomyosarcoma & 35 & 4.466 & 1.270 to 15.703 & 0.020 \\
Liposarcoma & 27 & 5.908 & 1.502 to 23.234 & 0.011 \\
Angiosarcoma & 23 & 1.648 & 0.558 to 4.869 & 0.366 \\
Synovialsarcoma & 18 & 8.289 & 1.321 to 52.016 & 0.024 \\
MPNST* & 9 & - & & \\
Carcinosarcoma* & 4 & - & & \\
Chondrosarcoma* & 3 & - & & \\
Rhabdomyosarcoma* & 3 & - & & \\
malignant SFT* & 2 & - & & \\
Alveolar soft tissue sarcoma* & 2 & - & & \\
Myxofibrosarcoma* & 2 & - & & \\
\hline *Number of patients too small for Kaplan-Meier analysis. &
\end{tabular}

To prove that the increase of $p 16$ mRNA went along with a higher expression on the protein level, U2OS cells, treated for $72 \mathrm{~h}$ using different 5-Aza concentrations, were lysed and subjected to western blot. This revealed a dose-dependent increase of p16 protein, indicating that this effect was mediated by the demethylation of the $p 16$ gene (figure 3D).

\section{DISCUSSION}

This study demonstrates the absence of p16(INK4a) expression in a subgroup of high-grade sarcomas with a very aggressive malignant potential in a well-characterised cohort of 183 soft tissue and bone sarcomas. To our knowledge, this is the first study to show that the absence of $\mathrm{p} 16$ (INK4a) expression correlates with reduced patient survival in sarcomas. This is particularly evident in oncogene/translocation-associated sarcomas, for example, dedifferentiated liposarcomas and synovial sarcomas $(\mathrm{p}<0.001$ and $\mathrm{p}=0.010$, respectively), indicating an upregulation of $\mathrm{p} 16(\mathrm{INK} 4 \mathrm{a})$ in the initial phase of tumour progression
Figure 3 The $p 16$ (INK4a) promoter is methylated in U2OS cells: (A) MSP analysis of the $p 16$ promoter in different sarcoma cell lines. $M$ and $U$ represent methylated or unmethylated alleles, respectively. Water was used as template for technical negative control reaction and (bisulphite treated) DNA from the colorectal cancer cell line HCT116 as positive control for both primer sets. (B) MSP analysis of the p16 promoter in U2OS cells following treatment with different 5-Aza concentrations for 72 h. (C) qPCR analysis for the detection of $p 16$ mRNA upon treatment with 5-Aza, results were normalised to GAPDH mRNA. p16 mRNA levels of the cervix carcinoma cell line HeLa served as a positive control. Data are represented as mean $\pm S D(n=3)$. (D) Western blot analysis of p16 protein levels in U2OS cells treated for 72 using different 5-Aza concentrations. $\alpha$-tubulin served as a loading control.

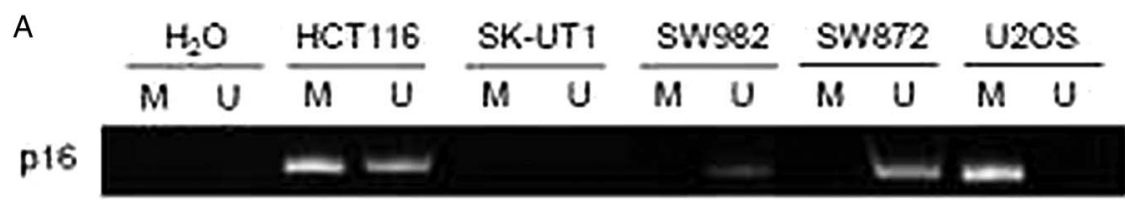

B U2OS 72 \& 5-Aza

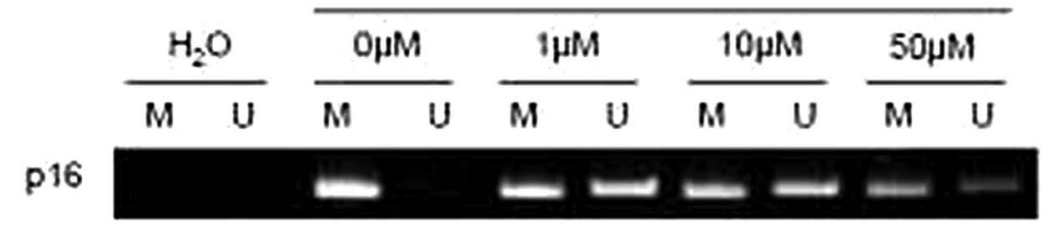

C

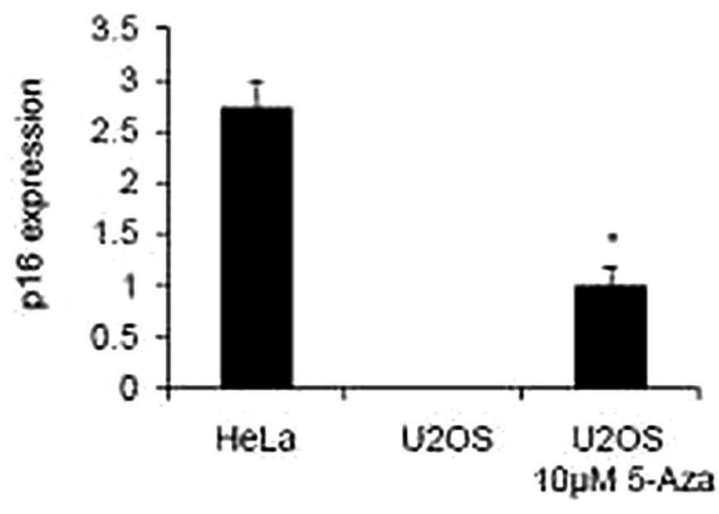

D

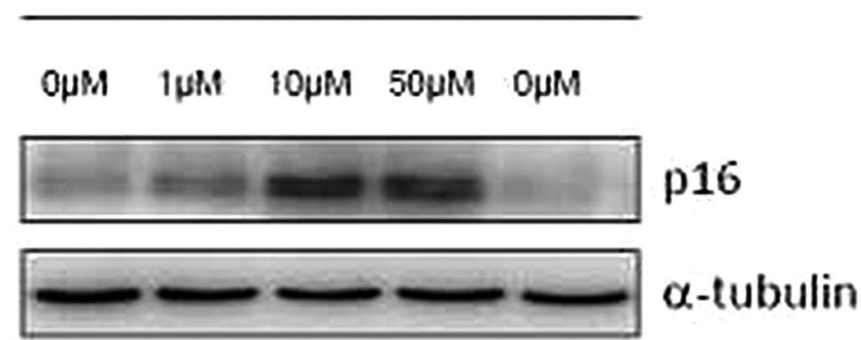


Figure 4 Modell of oncogeneinduced senescence (OIS) in liposarcoma genesis. In human liposarcomas high p16(INK4a) expression is found in welldifferentiated liposarcomas (WDLS) induced by initiated MDM2/CDK4 amplification indicating a senescence barrier. Loss of p16(INK4a) is observed in a subgroup of dedifferentiated liposarcomas in the final phase of tumour progression with significantly reduced patient survival.

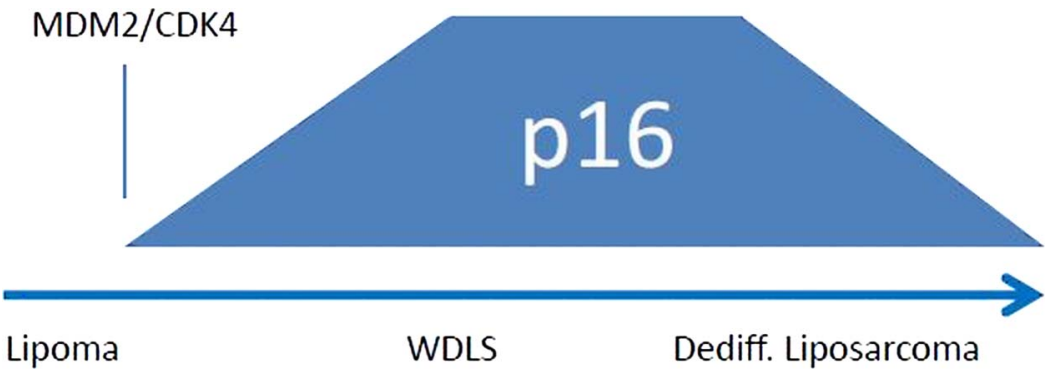

as a senescence barrier, and a loss of p16(INK4a) expression in the final phase of tumour progression.

Our observations are in concordance with investigations concerning human naevi where the concept of oncogene-induced senescence (OIS) has been established. ${ }^{6}{ }^{13}$ In melanocytic naevi, a stop of cell proliferation is correlated with a high p16INK4a expression and frequently found in combination with mutations in the BRAF oncogene. In the model proposed by Michaloglou et al, ${ }^{13}$ BRAF-signaling primarily prompts benign naevi to exhibit a limited growth due to an induction of senescence. This senescence barrier must be overcome by further genetic alterations in order to lead to the development of malignant melanoma. Previously, we showed that upregulation and downregulation of $\mathrm{p} 16$ (INK4a) in BRAF-mutated polyps/adenomas indicated a senescence barrier in the serrated route of colonic cancer. ${ }^{6}$ In this study, we expanded the analysis to mesenchymal neoplasms. Our data indicate an analogous process in human sarcomas where oncogene-induced senescence driven by initiating amplifications or translocations of distinct sarcoma types might cause a growth arrest and stop further malignant transformation. The loss of p16 (INK4a) expression might overrun the senescence barrier and could enable further progression into a very aggressive malignant subtype of sarcoma (figure 4).

We observed p16(INK4a) expression in liposarcoma (88.9\%), leiomyosarcoma (85.7\%), synovial sarcoma (77.8\%), undifferentiated pleomorphic sarcoma (UPS) (69.1\%), and angiosarcoma (60.9\%). However, in UPS and in angiosarcomas, no correlation between p16(INK4a) expression and observed survival was noted. This is in concordance with the theory that undifferentiated tumours have such a complex and disturbed karyotype and/ or protein pattern, that no regulated pathways exist. The senescence mechanism might not depend on regulated pathways in these kinds of tumours, and the regulation of the cell interaction might be highly disconnected and chaotic. On the other hand, the so-called oncogene or translocation-depending sarcomas have regulated pathways in tumour progression at least in the early phase of malignant transformation with robust biomarkers. Interestingly, p16(INK4a) expression was very recently described as a robust diagnostic biomarker for well-differentiated liposarcomas (WDLS) distinguishing WDLS from lipomas in combination or alone with the immunohistochemical markers MDM2 and CDK4. ${ }^{14} 15$ Our results confirm these findings, all WDLS show a senescence-induced high p16(INK4a) expression. Furthermore, high expression of p16(INK4a) is an interesting diagnostic biomarker in leiomyosarcomas that has not been described before. However, further studies are needed to confirm these findings.

Demethylation tests with p16(INK4a) in four sarcoma cell lines demonstrated that on mRNA level, two out of four cell lines showed expression of $\mathrm{p} 16$ (INK4a). In one remaining sarcoma cell line, p16(INK4a) expression could be restored after a demethylation test. This in vitro study showed that downregulation of p16(INK4a) may be explained by DNA hypermethylation in sarcoma cells. This is in concordance with earlier studies and might be at least one potential pathomechanism of the absence for p16(INK4a). However, alternative mechanisms do exist. The cell line, SK-UT1, showed no methylated or unmethylated alleles, and may have undergone a deletion of the gene as one possible alternative mechanism to silence the p16(INK4a) expression.

p16(INK4a) Is an inhibitor of the cyclin-dependent kinases $(\mathrm{CDK})$, which phosphorylate the retinoblastoma $(\mathrm{Rb})$ protein, a classic tumour suppressor and key component of the G1/S checkpoint. Interestingly, very recently, Dickson et $a l^{16}$ published a phase II trial of the CDK4 inhibitor, PD0332991, in patients with an advanced CDK4-amplified well-differentiated or dedifferentiated liposarcoma. The selective cyclin-dependent kinase 4 (CDK4)/CDK6 inhibitor PD0332991 inhibits growth and induces senescence in cell lines and xenografts. Dickson et al were able to demonstrate that treatment with a CDK4 inhibitor was associated with a favourable progression-free survival rate in patients with CDK4-amplified and RB-expressing WDLS/DDLS who had experienced progressive disease despite standard systemic therapy. Since p16(INK4a) is also a CDK4 inhibitor, these observations are in line with our results and according to our observations, CDK4 inhibitors obviously induce senescence in adipocytic differentiated tumours, and also in other mesenchymal-malignant neoplasms.

In conclusion, we illustrate in this study that upregulation of $\mathrm{p} 16$ (INK4a) is associated with the induction of oncogene-induced senescence, and indicates a senescence barrier. Downregulation of p16(INK4a) is significantly correlated with reduced patient survival in dedifferentiated liposarcomas, synovial sarcomas and leiomyosarcomas and one possible mechanism is epigenetic silencing through aberrant methylation of the CDKN2A promotor. Further studies are needed to explore the functional role of p16(INK4a) and other cell-cycle regulators in the genesis of mesenchymal neoplasia. CDK inhibitor therapy might be a promising avenue to treat patients with these subtypes of sarcomas.

\section{Take home messages}

- p16(INK4a) is upregulated in sarcomas, and shows a distinct expression pattern in distinct subgroups.

- Reduced expression of p16(INK4a) is significantly associated with shorter patients survival in sarcomas.

- p16(INK4a)-negative sarcomas might indicate a tumour subgroup eligible for a more aggressive treatment.

- Downregulation of p16(INK4a) could be explained by DNA hypermethylation. 
Contributors TK: writing, design, immunhistochemistry AAH: statistical analysis, LL and RI: clinical data EK, SG and GS: clinical data update, writing HHand HS: cell line experiments TKi: Design, data collection.

\section{Competing interests None.}

Provenance and peer review Not commissioned; externally peer reviewed.

\section{REFERENCES}

1 Fletcher CDM, World Health Organization, International Agency for Research on Cancer. WHO classification of tumours of soft tissue and bone. 4th ed. Lyon: IARC Press, 2013:468.

2 Sorbye SW, Kilvaer TK, Valkov A, et al. Prognostic impact of Jab1, p16, p21, p62, Ki67 and Skp2 in soft tissue sarcomas. PloS one 2012;7:e47068.

3 Knosel T, Heretsch S, Altendorf-Hofmann A, et al. TLE1 is a robust diagnostic biomarker for synovial sarcomas and correlates with $t(X ; 18)$ : analysis of 319 cases. Eur I Cancer 2010;46:1170-6.

4 Bennecke M, Kriegl L, Bajbouj M, et al. Ink4a/Arf and oncogene-induced senescence prevent tumor progression during alternative colorectal tumorigenesis. Cancer cell 2010;18:135-46.

5 Collado M, Blasco MA, Serrano M. Cellular senescence in cancer and aging. Cell 2007;130:223-33.

6 Kriegl L, Neumann J, Vieth M, et al. Up and downregulation of p16(Ink4a) expression in BRAF-mutated polyps/adenomas indicates a senescence barrier in the serrated route to colon cancer. Mod Pathol 2011;24:1015-22.

7 Dankort $D$, Filenova $E$, Collado $M$, et al. A new mouse model to explore the initiation, progression, and therapy of BRAFV600E-induced lung tumors. Genes Dev 2007;21:379-84.
8 Gutierrez-Reyes G, del Carmen Garcia de Leon M, Varela-Fascinetto G, et al. Cellular senescence in livers from children with end stage liver disease. PloS one 2010:5:e10231.

9 Jeong J, Park YN, Park JS, et al. Clinical significance of p16 protein expression loss and aberrant p53 protein expression in pancreatic cancer. Yonsei Med J 2005;46:519-25.

10 Gonzalez-Quevedo R, Garcia-Aranda C, Moran A, et al. Differential impact of p16 inactivation by promoter methylation in non-small cell lung and colorectal cancer: clinical implications. Int I Oncol 2004;24:349-55.

11 Issels RD, Lindner LH, Verweij J, et al. Neo-adjuvant chemotherapy alone or with regional hyperthermia for localised high-risk soft-tissue sarcoma: a randomised phase 3 multicentre study. Lancet Oncol 2010;11:561-70.

12 Knosel T, Emde A, Schluns K, et al. Immunoprofiles of 11 biomarkers using tissue microarrays identify prognostic subgroups in colorectal cancer. Neoplasia 2005; 7:741-7.

13 Michaloglou C, Vredeveld LC, Soengas MS, et al. BRAFE600-associated senescence-like cell cycle arrest of human naevi. Nature 2005;436:720-4.

14 Gonzalez RS, McClain CM, Chamberlain BK, et al. Cyclin-dependent kinase inhibitor 2A ( $p 16)$ distinguishes well-differentiated liposarcoma from lipoma. Histopathology 2013;62:1109-11.

15 Thway K, Flora R, Shah C, et al. Diagnostic utility of p16, CDK4, and MDM2 as an immunohistochemical panel in distinguishing well-differentiated and dedifferentiated liposarcomas from other adipocytic tumors. Am I Surg Pathol 2012;36:462-9.

16 Dickson MA, Tap WD, Keohan ML, et al. Phase II trial of the CDK4 inhibitor PD0332991 in patients with advanced CDK4-amplified well-differentiated or dedifferentiated liposarcoma. J Clin Oncol 2013;31:2024-8. 


\section{$\mathrm{JCP}$}

Loss of p16(INK4a) is associated with reduced patient survival in soft tissue tumours, and indicates a senescence barrier

Thomas Knösel, Annelore Altendorf-Hofmann, Lars Lindner, Rolf Issels, Heiko Hermeking, Gesa Schuebbe, Sebastian Gibis, Helge Siemens, Eric Kampmann and Thomas Kirchner

J Clin Pathol 2014 67: 592-598 originally published online April 19, 2014 doi: 10.1136/jclinpath-2013-202106

Updated information and services can be found at:

http://jcp.bmj.com/content/67/7/592

These include:

Supplementary Supplementary material can be found at: Material http://jcp.bmj.com/content/suppl/2014/04/22/jclinpath-2013-202106.D

References This article cites 15 articles, 2 of which you can access for free at: http://jcp.bmj.com/content/67/7/592\#BIBL

Email alerting Receive free email alerts when new articles cite this article. Sign up in the service box at the top right corner of the online article.

Topic Articles on similar topics can be found in the following collections Collections

Colon cancer (231)

\section{Notes}

To request permissions go to:

http://group.bmj.com/group/rights-licensing/permissions

To order reprints go to:

http://journals.bmj.com/cgi/reprintform

To subscribe to BMJ go to:

http://group.bmj.com/subscribe/ 